\title{
Distinct Features of Psychosocial Distress of Adolescents and Young Adults with Cancer Compared to Adults at Diagnosis: Patient-Reported Domains of Concern
}

\author{
Alannah Smrke, MD, ${ }^{1}$ Bonnie Leung, MN, ${ }^{1}$ Amirrtha Srikanthan, MD, MHSc, ${ }^{2}$ \\ Melanie McDonald, MSW, ${ }^{3}$ Alan Bates, MD, PhD, ${ }^{3}$ and Cheryl Ho, MD ${ }^{1}$
}

\begin{abstract}
Purpose: Adolescents and young adults (AYA) (18-40) are a population of patients with cancer, who have distinctive developmental and psychosocial pressures. Using validated distress screening tools, we investigated psychosocial needs of AYA compared to older adults with cancer at diagnosis.

Methods: AYA and older adult patients from British Columbia, Canada, between 2011 and 2016, who completed the Canadian Problem Checklist (CPC) and the PsychoSocial Screen for Cancer-Revised (PSSCAN-R) within 6 months of their cancer diagnosis were included in the study. Emotional, informational, physical, practical, social, and spiritual domain concerns are identified using the CPC. Psychosocial needs and distress are evaluated using the PSSCAN-R. Baseline demographics were obtained from the cancer registry. Based on gender, primary tumor site, and presence of metastasis, a 3:1 case match was performed with older adults (>40 years old). Statistical analyses included Chi square and Fisher's exact tests.
\end{abstract}

Results: Two thousand and forty five AYA were case matched with 6050 older adults. Majority of patients were female $(61.9 \%)$, and at diagnosis, $12.1 \%$ had metastatic disease. Top three tumor types were breast (20.4\%), lymphoma (11.5\%), and gastrointestinal (10.8\%). The top five concerns for AYA (\% AYA, \% adults) were fear/worry $(56.6,42.9)$, understanding of illness $(47.6,41.4)$, sleep $(35.2,28.9)$, sadness $(34.1,20.0)$, and finances $(33.8,15.0)$. AYA reported higher symptoms of anxiety at baseline (\% AYA, $\%$ older adults), both moderate $(26.0,19.9)$ and severe $(26.6,17.1) p<0.01$.

Conclusion: Significant differences in psychosocial needs for AYA were seen at diagnosis across multiple domains, specifically, higher emotional, informational, physical, and financial distress. Development of supportive programming geared toward these domains early at diagnosis could benefit this distinct population.

Keywords: anxiety, depression, psychosocial needs, distress, cancer diagnosis

\section{Background}

A DOLESCENTS AND YOUNG adults (AYA) represent a small, but unique group of patients. A cancer diagnosis at this time alters a young adult's normal trajectory of development, often causing long-lasting effects that persist into later life. With the number life years affected by cancer highest in AYA populations ${ }^{1}$ and the improving outcomes of cancer therapy, there needs to be renewed attention to the psychosocial and developmental impacts of a cancer diagnosis and treatment in young people.
Cancer treatments are often intensive, and this has more considerable practical implications for AYA, given they are at a different place in career and personal growth. ${ }^{2}$ Navigating the medical system and self-advocacy is new for many AYA, likely adding further distress to a life-altering diagnosis. ${ }^{2}$ Long-term survivors can experience increased somatic complaints, academic difficulties, and more worry around health-related concerns. ${ }^{3}$

There is extensive literature showing distress among adult cancer patients, with $35 \%-45 \%$ experiencing significant levels. ${ }^{4}$ At $4-12$ months following diagnosis, $27 \%-28 \%$ of

\footnotetext{
${ }^{1}$ Medical Oncology, BC Cancer, Vancouver Centre, Vancouver, Canada.

${ }^{2}$ Division of Medical Oncology, The Ottawa Hospital Cancer Centre, Ottawa, Canada.

${ }^{3}$ Psychosocial Oncology, BC Cancer, Vancouver Centre, Vancouver, Canada.
} 
AYA also exhibit clinically significant psychological distress. $^{5,6}$ This distress remained high over a 12-month period when adjusted for treatment and work/employment status. ${ }^{6}$ There is evidence that among AYA, distress can lead to reduced treatment compliance, ${ }^{7}$ which may correlate with poorer health outcomes in this vulnerable population. Although AYA needs have been studied at different time points on their illness trajectory, ${ }^{5,6,8-10}$ there is limited understanding of distress experienced by AYA at diagnosis compared to older adults.

The purpose of this study is to characterize the unique features of AYA distress compared to that of adults with similar cancers and to understand the drivers of their distress using the Canadian Problem Checklist (CPC) and a validated screening questionnaire (PsychoSocial Screen for CancerRevised [PSSCAN-R]).

\section{Methods}

A retrospective review of all AYA patients who completed the CPC and PSSCAN-R at BC Cancer within 6 months of their cancer diagnosis between 2011 and 2016 was conducted. Patients $<18$ years of age were treated at BC Children's Hospital and were excluded from our study. BC Cancer is a provincial cancer program within Canada that serves a population of 4.6 million. Baseline demographics were obtained through the cancer registry and discrepancies were abstracted by chart review. Nonmetastatic disease was defined as local or locoregional disease and metastatic disease included all patients with distant spread at the time of completion of the screening questionnaires. Community size was estimated from current postal code and the Statistics Canada Postal Code Conversion File Plus Version 6D was used to estimate household income.

The modified Social Network and Support Assessment tool used in the Epidemiological Study of the Elderly ${ }^{11}$ was used for social support assessment. The patients are asked dichotomous items, including whether they live alone, have emotional support, and have regular contact with friends and family.

The CPC was generated from a Screening for Distress Toolkit Working Group. ${ }^{4}$ Patients are asked to select which, with multiple responses permitted, of the evidence-based list of common concerns or problems ${ }^{12}$ they had experienced within the past week. These concerns were grouped into the following domains: emotional, informational, practical, spiritual, social, and physical.

PSSCAN-R has been developed and validated as a tool to assess distress in patients with cancer. ${ }^{13,14}$ The Hospital Anxiety and Depression Scale ${ }^{9}$ was used to validate the PSSCAN-R to enable identification of subclinical and clinical symptoms of anxiety and depression. The selected scores are optimized to achieve the best sensitivity and specificity for identification of patients with distress. Briefly, levels of distress with specified time points were assessed on a Likert Scale (Not at all " 0 " to very much " 5 "), and mean scores were calculated and divided into low, ${ }^{1-7}$ moderate ${ }^{8-10}$ and severe $(>10)$ symptoms of anxiety or depression. ${ }^{13,14}$

\section{Statistical analysis}

A 3:1 case match based on gender, primary tumor site, and metastatic status was performed with older adults ( $>40$ years old) and AYA (18-40 years old). Univariate analysis was performed using Chi square and Fisher's exact tests. The $p$-value of $<0.05$ was used to define statistical significance. Statistical analyses were conducted using IBM SPSS Statistics Software, version 25 (SPSS Institute, Inc., Cary, NC).

\section{Ethics statement}

This study was pre-approved by the BC Cancer Ethics Board and privacy office (University of British ColumbiaBC Cancer Research Ethics Board; H16-01047). Consent was not obtained from individual patients; however, patient data were de-identified for analysis by the investigators.

\section{Results}

\section{Baseline characteristics}

There were 2045 patients diagnosed between ages 18 and 39 between 2011 and 2016, who completed the CPC and PSSCAN-R (Table 1) at the time of diagnosis. Approximately $61.9 \%$ of patients were female. The most common tumor types were breast $(20.4 \%)$ and gastrointestinal $(10.7 \%)$. The majority $(85.6 \%)$ were nonmetastatic at the time of referral, while $12.1 \%$ were metastatic, with $2.3 \%$ unknown. Majority of AYA and adults did not live alone and had regular contact with friends and family. Over half of both cohorts were from large cities (population $>1.5$ million). Incomes were distributed; however, slightly more AYA were low to mid quintile, compared to slightly more older adults in mid-upper to upper quintile.

\section{Psychosocial domains (CPC)}

The top six specific areas of distress for AYA (\% AYA, \% older adults) were fear/worry $(56.6,42.9)$ understanding of illness $(47.6,41.4)$, sleep $(35.2,28.9)$, sadness $(34.1,20.0)$, finances $(33.8,15.0)$, and school/work $(33.7,10.5)$. Differences between groups were all statistically significant $(p<0.01)$ (Fig. 1).

The greatest differences compared to older adults (\% AYA, $\%$ older adults) were work/school $(33.7,10.5)$, finances $(33.8$, $15)$, fear/worry $(56.6,42.9)$, sadness $(34.1,20.4)$, frustration/anger $(32.8,20.0)$, worry about family $(33.3,24.0)$, and intimacy/sexuality $(13.2,5.3)$. The subdomain 'getting to appointment' $(9.2 \%$ AYA and $11.2 \%$ adult, $p<0.01)$ was the only subdomain more often reported in adults than AYA.

\section{Anxiety and depression screening scores}

Based on PSSCAN-R scoring classification, more AYA than older adults (\% AYA, \% older adults) had moderate $(26.0,19.9)$ or severe $(26.6,17.1)$ symptoms of anxiety $(p<0.01)$. The median scores for symptoms of anxiety were AYA 8 [interquartile range (IQR) 5.5-11] and older 6 (IQR 5-9) (Fig. 2).

The majority of depression symptom scores were low in the AYA (67.7\%) and older adult (73.6\%) population. More AYA (\% AYA, \% older adults) had moderate $(19.2,15.3)$ or severe $(13.1,11.1)$ symptoms of depression scores than older adults $(p<0.01)$. The median scores for symptoms of depression were AYA 6 (IQR 5-8) and older 5 (IQR 5-7).

Suicidal ideation was low and similar in the AYA cohort compared to older adults. The incidence of significant 
Table 1. Baseline Characteristics

\begin{tabular}{|c|c|c|c|}
\hline & $\begin{array}{l}\text { Case match adult group } \\
\qquad \mathrm{N}=8180\end{array}$ & $\begin{array}{c}A Y A \\
\mathrm{~N}=2045\end{array}$ & $\%$ \\
\hline \multicolumn{4}{|l|}{ Matched variables } \\
\hline \multicolumn{4}{|l|}{ Age } \\
\hline Median age (IQR) & $65(55-74)$ & $33(28-37)$ & \\
\hline \multicolumn{4}{|l|}{ Age } \\
\hline $18-24$ & & 275 & 13.4 \\
\hline $25-29$ & & 370 & 18.1 \\
\hline $30-34$ & & 557 & 27.2 \\
\hline $35-39$ & & 843 & 41.2 \\
\hline \multicolumn{4}{|l|}{ Sex } \\
\hline Female & 3743 & 1266 & 61.9 \\
\hline Male & 2307 & 779 & 38.1 \\
\hline \multicolumn{4}{|l|}{ Tumor type } \\
\hline Breast & 1251 & 417 & 20.4 \\
\hline Cervix/endometrial & 486 & 162 & 8.0 \\
\hline Gastrointestinal & 654 & 218 & 10.8 \\
\hline Lymphoma & 696 & 232 & 11.5 \\
\hline Melanoma & 216 & 72 & 3.6 \\
\hline CNS & 365 & 123 & 6.0 \\
\hline Sarcoma & 411 & 137 & 6.8 \\
\hline Thyroid & 621 & 234 & 10.6 \\
\hline Other & 1350 & 450 & 22.2 \\
\hline Testes & & 227 & 11.1 \\
\hline Renal & & 77 & 3.8 \\
\hline Ovarian & & 76 & $\begin{array}{l}5.0 \\
3.7\end{array}$ \\
\hline Lung & & 23 & 1.1 \\
\hline Salivary & & 23 & 1.1 \\
\hline \multicolumn{4}{|l|}{ Stage at diagnosis } \\
\hline Metastatic & 744 & 248 & 12.1 \\
\hline Nonmetastatic & 5250 & 1750 & 85.6 \\
\hline \multirow[t]{2}{*}{ Unknown } & 141 & 47 & 2.3 \\
\hline & & & p Value \\
\hline \multicolumn{4}{|l|}{ Unmatched variables } \\
\hline \multicolumn{4}{|l|}{ Lives alone } \\
\hline Yes & & 269 & 13.3 \\
\hline No & & 1749 & 86.7 \\
\hline \multicolumn{4}{|c|}{ Has regular contact with friends and family } \\
\hline Yes & $5764(97.4 \%)$ & $1987(98.4 \%)$ & $<0.01$ \\
\hline No & $155(2.6 \%)$ & $32(1.6 \%)$ & \\
\hline \multicolumn{4}{|l|}{ Community size } \\
\hline$>1.5$ million & $3241(55.5 \%)$ & $1207(63.5 \%)$ & $<0.01$ \\
\hline $100,000-1.5$ million & $945(16.2 \%)$ & $292(15.4 \%)$ & $<0.01$ \\
\hline$<100,000$ & $1657(28.4 \%)$ & $2060(26.6 \%)$ & $<0.01$ \\
\hline \multicolumn{4}{|l|}{ Income } \\
\hline Lowest to mid lowest quintile & $2250(39.2 \%)$ & $774(42.0 \%)$ & $<0.01$ \\
\hline Middle quintile & $1065(18.5 \%)$ & $387(21.0 \%)$ & $<0.01$ \\
\hline Mid-upper to upper quintile & $2428(42.3 \%)$ & $680(36.9 \%)$ & $<0.01$ \\
\hline
\end{tabular}

IQR, interquartile range; AYA, adolescents and young adults; CNS, central nervous system.

suicidal thoughts ("quite a bit" or "very much so") was also low and not statistically different between cohorts.

\section{Discussion}

Our study highlights differences between AYA and their adult peers with respect to baseline distress and areas of concern at cancer diagnosis. Case matching was performed to reduce confounders and to assess the differential needs of AYA compared to adults. Understanding AYA needs at diagnosis will inform program development for AYA at a vulnerable time, with the hope that early engagement and support will translate into improved compliance and overall well-being. ${ }^{2,7,15}$

Our study consists of a Canadian population of predominantly young adults, with the majority of patients being older than 30 years. In our large cohort, $61.9 \%$ were females, which has been seen in other large cohorts, ${ }^{9}$ and reflects the gender distribution previously seen in AYA ${ }^{16-18}$ Ethnicity data were unavailable for our cohort, as this is not collected as part of the patient reported information and symptom measurement intake form. The effect of ethnicity on distress in our cohort is 
FIG. 1. Canadian Problem Checklist Domains reported by AYA and adults. (Black bar-AYA, White baradult). AYA, adolescents and young adults.

FIG. 2. PSSCAN-R Anxiety (A) and Depression (B) scores for AYA versus adults. PSSCAN-R, PsychoSocial Screen for CancerRevised.
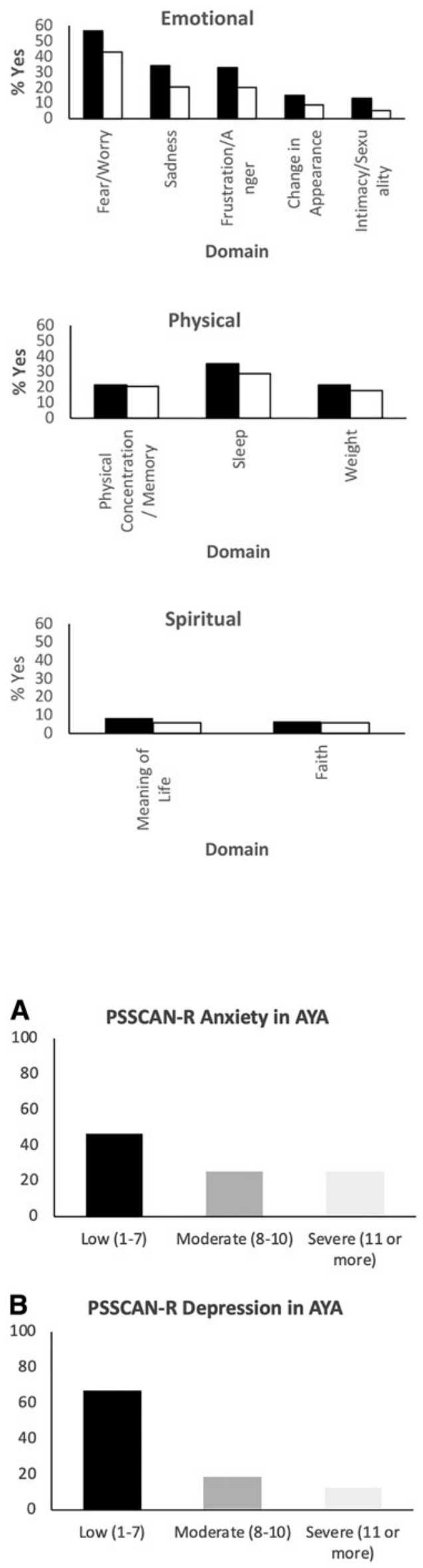
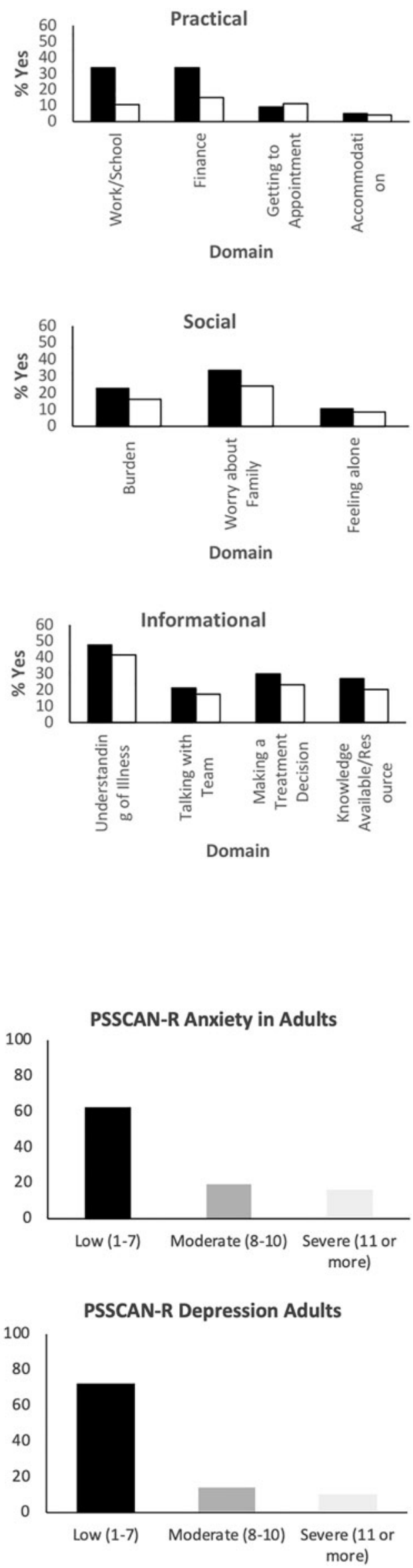
unknown. The top five most common cancers in our sample (breast, lymphoma, testes, gastrointestinal, and thyroid) are similar to other cohorts, with an older average age of AYA patients. ${ }^{9}$ Compared to worldwide incidence, less liver cancer ( $10.1 \%$ worldwide vs. $0.3 \%$ in our cohort) was seen, likely due to differing risk factors for hepatic disease in our North American population. Interestingly, cervical cancer incidence was similar to the world-wide incidence $(7.8 \%$ worldwide vs. $8.0 \%$ in our cohort). We expect Canadian incidence to decrease over time, as the human papillomavirus vaccine became publicly funded in 2006. ${ }^{19}$ Thus, comparison to other population-based studies suggests that the result of our study may be extrapolated to the general AYA population.

Across all domains, AYA experienced higher levels of concern than their matched adult peers. Concerns across emotional and practical domains were reported most often in AYA, while specific spiritual concerns were reported least often. It is well recognized that key areas of development are interrupted with a cancer diagnosis. ${ }^{2}$ Specifically, within evaluated domains, AYA expressed more concerns regarding work/school, finances, fear/worry, sadness, frustration/anger, worry about family, and intimacy/sexuality than their adult counterparts. Concerns regarding work, school, finances, and sexuality may have been more common to AYA as their cancer diagnosis occurs during an important personal developmental period. ${ }^{2}$ Our study supports that, compared to adults, important areas to focus program development on for AYA are practical concerns such as work/school and finances. Furthermore, our results support augmented resources to address higher levels of emotional needs for AYA at diagnosis. Although the absolute number is low, AYA were more than twice as likely to report intimacy/sexuality concerns, which is congruent with previous literature that sexual function can be uniquely affected in AYA with a cancer diagnosis. ${ }^{20}$ The level of concern within our cohort for sexual/intimacy is similar to other cohorts. ${ }^{5}$ Comparison to other cohorts is challenging due to different methodology and time points used to screen for distress in AYA across multiple studies. Our study does support results from other reports identifying informational and practical domains are important areas of need within the AYA population. ${ }^{5,10}$

In our cohort, AYA had higher rates of moderate or severe symptoms of anxiety or depression compared to adults. Over half of our AYA cohort had symptoms of anxiety and over a quarter had symptoms of depression; direct comparison to an AYA group without cancer is difficult. In a recent survey of 38,015 adolescents (age 12-17) in British Columbia, selfreported rates of anxiety/panic attacks (8\%-29\%) or depression $(10 \%-20 \%)$ were lower than our AYA population within the same province. ${ }^{21}$ Meta-analysis of 24 studies, including 4007 adults from multiple time points and care settings, identified up to $38.2 \%$ of adult patients have some form of emotional distress, with the prevalence of depression being $14.3 \%-18.5 \% .^{22}$ Our study noted higher levels of anxiety symptoms rather than depressive symptoms, which may relate to adjustment to a new diagnosis of cancer and immediate concerns about life changes.

Symptoms of anxiety and depression may evolve over the course of cancer treatment. In a study of 52 AYA (16-30 years old) in Australia, where surveys were taken within 4 months of diagnosis, $8 \%-15 \%$ of participants met criteria for anxiety using the State-Trait Inventory form Y, and 2\%$15 \%$ of participants met criteria for depression defined using the Beck Depression Index second edition fast screen. ${ }^{10}$ However, rates of depression were shown to increase over time. Using the General Anxiety Disorder-7, a cohort of German AYA $^{9}$ within 5 years of cancer diagnosis showed prevalence of moderate to severe anxiety of $4.6 \%-16.2 \%$, and moderate or severe depression scores using the Patient Health Questionnaire-9 were seen in $29.5 \%$ of patients. Their results reflect the differential rates of anxiety and depression, which exist along a treatment spectrum. Interestingly, rates of anxiety are higher in our cohort of AYA at diagnosis compared to the German and Australian cohort, but depression scores are higher in the German cohort of AYA surveyed within 5 years of diagnosis. More research is required as to whether this reflects the acute reaction in AYA being predominantly anxiety, with depression emerging as the predominant symptom over the longer term.

Although our study is large, there are limitations. The questionnaire was administered at one time point, and we do not have longitudinal data on how the distress of AYA changes over time compared to adults. The voluntary questionnaires were provided at the first consultation at our institution and patients may not have completed them if there was insufficient clinic time or they were unable to read available versions in English, French, traditional Chinese, or Punjabi. The case match analysis was unable to address all of the common AYA cancer diagnoses like testes cancer due the lower incidence in older populations. Importantly, fertility concerns are not captured in the CPC or PSSCAN-R, and this is a well-recognized concern for AYA. ${ }^{23}$ Finally, we did not capture whether AYA or adults had a pre-cancer psychiatric diagnosis. Despite these limitations, our study gives insight into a large population of older AYA patients' needs at the time of their cancer diagnosis and points to how best to assist in providing relevant support and resources.

At BC Cancer, our psychosocial oncology department offers a number of programs relevant to AYA patients, including mindfulness-based stress reduction, memory and attention adaptation training, and return to work and relaxation courses. Our Young Adults Group provides art therapy, which allows AYA emotional exploration and connection with peers. Our data suggest that significant numbers of AYA specifically express concerns regarding practical support and it is critical to connect them to available resources early. Recognizing the limitations of a single time point assessment of distress, BC Cancer has also initiated a project that will involve serial assessments of quality of life and distress throughout the cancer journey to become better partners in patient care. Our goal is to understand the needs of our AYA patients at diagnosis to improve treatment adherence and mitigate the long-term psychosocial toxicity of a cancer diagnosis and cancer treatment.

\section{Conclusion}

In our large cohort, there were notable differences in distress in AYA compared to their case-matched adult counterparts across multiple domains. AYA are more likely to have anxiety at diagnosis compared to adults, while rates of depression were low across both cohorts. Understanding distress around this time point is critical to development of 
AYA-specific programming, and augmenting support in key areas to ensure AYA comfort and engagement in a predominantly older adult-focused system.

\section{Author Disclosure Statement}

No competing financial interests exist.

\section{Funding Information}

This study was supported by the BC Cancer Foundation through funding of OaSIS.

\section{References}

1. Bleyer A, Ferrari A, Whelan J, Barr RD. Global assessment of cancer incidence and survival in adolescents and young adults. Pediatr Blood Cancer. 2017;64(9):e26497.

2. Zebrack B, Butler M. Context for understanding psychosocial outcomes and behavior among adolescents and young adults with cancer. J Natl Compr Cancer Netw. 2012;10(9):1151-6.

3. Zeltzer LK. Cancer in adolescents and young adults psychosocial aspects. Long-term survivors. Cancer. 1993; 71(S10):3463-8.

4. Bultz BD, Groff SL, Fitch M, et al. Implementing screening for distress, the 6th vital sign: a Canadian strategy for changing practice. Psychooncology. 2011;20(5):463-9.

5. Zebrack BJ, Corbett V, Embry L, et al. Psychological distress and unsatisfied need for psychosocial support in adolescent and young adult cancer patients during the first year following diagnosis. Psychooncology. 2014;23(11): 1267-75.

6. Kwak M, Zebrack BJ, Meeske KA, et al. Trajectories of psychological distress in adolescent and young adult patients with cancer: a 1-year longitudinal study. J Clin Oncol. 2013;31(17):2160-6.

7. Butow P, Palmer S, Pai A, Goodenough B, Luckett T, King M. Review of adherence-related issues in adolescents and young adults with cancer. J Clin Oncol. 2010;28(32): 4800-9.

8. Zebrack BJ, Block R, Hayes-Lattin B, et al. Psychosocial service use and unmet need among recently diagnosed adolescent and young adult cancer patients. Cancer. 2013; 119(1):201-14.

9. Geue K, Brähler E, Faller H, et al. Prevalence of mental disorders and psychosocial distress in German adolescent and young adult cancer patients (AYA). Psychooncology. 2018;27(7):1802-9.

10. Dyson GJ, Thompson K, Palmer S, et al. The relationship between unmet needs and distress amongst young people with cancer. Support Care Cancer. 2012;20(1):75-85.

11. Seeman TE, Berkman LF. Structural characteristics of social networks and their relationship with social support in the elderly: who provides support. Soc Sci Med. 1988; 26(7):737-49.
12. Ashbury FD, Findlay H, Reynolds B, McKerracher K. A Canadian survey of cancer patients' experiences: are their needs being met? J Pain Symptom Manage. 1998;16(5): 298-306.

13. Linden W, Vodermaier AA, McKenzie R, et al. The Psychosocial Screen for Cancer (PSSCAN): further validation and normative data. Health Qual Life Outcomes. 2009; 7(1):16.

14. Linden W, Yi D, Barroetavena MC, et al. Development and validation of a psychosocial screening instrument for cancer. Health Qual Life Outcomes. 2005;3(1):54.

15. Zebrack B, Isaacson S. Psychosocial care of adolescent and young adult patients with cancer and survivors. J Clin Oncol. 2012;30(11):1221-6.

16. Howlader NNA, Krapcho M, Miller D, et al. (Eds). SEER cancer statistics review, 1975-2016. Bethesda, MD: National Cancer Institute; 2019.

17. Canadian Cancer Society SC. Canadian Cancer Statistics. Toronto, ON: Canadian Cancer Statistics Advisory Committee; 2019.

18. Fidler MM, Gupta S, Soerjomataram I, et al. Cancer incidence and mortality among young adults aged 20-39 years worldwide in 2012: a population-based study. Lancet Oncol. 2017;18(12):1579-89.

19. Bird Y, Obidiya O, Mahmood R, et al. Human papillomavirus vaccination uptake in Canada: a systematic review and meta-analysis. Int J Prev Med. 2017;8:71.

20. Wettergren L, Kent EE, Mitchell SA, et al. Cancer negatively impacts on sexual function in adolescents and young adults: the AYA HOPE study. Psychooncology. 2017; 26(10):1632-9.

21. Society MC. Balance and connection in BC: The health and well-being of our youth. Results of the 2018 BC Adolescent Health Survey Vancouver, BC: McCreary Centre Society; 2019.

22. Mitchell AJ, Chan M, Bhatti H, et al. Prevalence of depression, anxiety, and adjustment disorder in oncological, haematological, and palliative-care settings: a metaanalysis of 94 interview-based studies. Lancet Oncol. 2011; 12(2):160-74.

23. Murphy D, Klosky JL, Reed DR, et al. The importance of assessing priorities of reproductive health concerns among adolescent and young adult patients with cancer. Cancer. 2015;121(15):2529-36.

Address correspondence to:

Cheryl Ho, MD

Medical Oncology

$B C$ Cancer

Vancouver Centre

600 West 10th Avenue

Vancouver, BC V5Z4E6

Email: cho@bccancer.bc.ca 